Europe PMC Funders Group

Author Manuscript

Nature. Author manuscript; available in PMC 2013 February 07.

Published in final edited form as:

Nature. ; 482(7386): 474-475. doi:10.1038/482474a.

\title{
CELL BIOLOGY:
}

\section{Collagen secretion explained}

\author{
David J. Stephens \\ Cell Biology Laboratories, School of Biochemistry, University of Bristol, Bristol BS8 1TD, UK. \\ david.stephens@bristol.ac.uk
}

\section{Abstract}

Cells package proteins into vesicles for secretion to the extracellular milieu. A study shows that an enzyme modifies the packaging machinery to encapsulate unusually large proteins such as collagen.

Together with other extracellular proteins, collagen provides the structural framework (or matrix) onto which tissues develop and function. It is synthesized as a rigid rod precursor (procollagen) of about 300 nanometres in length in the endoplasmic reticulum, an intracellular organelle. Procollagen - like nearly all secreted proteins - is then packaged into transport vesicles for delivery to another organelle, the Golgi apparatus, before its secretion to the cell's surroundings. Transport vesicles, however, are typically less than 100 $\mathrm{nm}$ in size, as they are generated from the endoplasmic reticulum by a group of proteins (the COPII coat) that co-assemble as a structurally defined polyhedral cage ${ }^{1}$. On page XX of this issue, Jin et al. ${ }^{2}$ reveal that modification of one of the COPII proteins enables formation of vesicles that are large enough to hold procollagen.

The outer layer of the COPII coat is assembled using structural elements comprised of the proteins Sec13 and Sec31 (Fig. 1a). Although it is thought that the hinges between these elements have sufficient flexibility to allow formation of vesicles of various sizes ${ }^{3,4}$, very little was known about how vesicle size could be controlled. Jin and colleagues ${ }^{2}$ show that Sec31 can be modified by ubiquitination - the attachment of one or several copies of the small protein ubiquitin. Although ubiquitination can 'mark' a protein for degradation, it is becoming increasingly clear that it can also affect protein function ${ }^{5}$

Specifically, the authors ${ }^{2}$ report that the enzyme $\mathrm{Cul}^{\mathrm{Klhl} 12}$ adds a single ubiquitin to a small pool of Sec31 molecules, and that this modification is required to drive the secretion of collagen from mouse cells. Using high-resolution electron microscopy, they found that cells that overexpression of $\mathrm{Cul}^{\mathrm{Klhl1}}{ }^{2}$ produced large COPII structures, up to $500 \mathrm{~nm}$ in diameter - sufficient to accommodate procollagen. The simplest explanation for these observations is that ubiquitin attachment to $\mathrm{Sec} 31$ results in a structural change in the COPII cage that alters coat flexibility and thus allows the encapsulation of procollagen in a nascent vesicle (Fig. 1b).

Jin and colleagues' observation that only some Sec31 molecules are modified strongly indicates that the addition of ubiquitin does not directly modulate the mechanics of COPII coat assembly. Instead, Sec31 ubiquitination might lead to recruitment of an additional, unknown protein that would perform this role - for example, by further stabilizing lateral Sec13-Sec31 interactions. Identification of this additional factor and a more detailed molecular explanation of the modified geometry of the vesicle coat are challenges that need to be addressed in the future. 
Ubiquitination of some Sec31 molecules could be an ongoing process that facilitates the formation of large COPII vesicles as a routine cell function or, alternatively, large vesicles might be formed only on demand. In the latter case, however, it is not immediately obvious how $\mathrm{Cul}^{\mathrm{K}}{ }^{\mathrm{Kl} 112}$, located in the cytoplasm, would sense the presence of newly synthesized procollagen, which is found within the endoplasmic reticulum. A potential candidate for relaying this information across the endoplasmic reticulum membrane is the transmembrane protein TANGO1, which forms part of a packaging receptor that is essential for procollagen secretion ${ }^{6,7}$. TANGO1, however, does not contact Sec31 directly, nor is it packaged into fully formed vesicles, and so its possible connection to $\mathrm{Cul}^{\mathrm{Klhl}}{ }^{12}$ remains unclear.

Other key questions remain. Does collagen become entirely encapsulated in a large COPII cage during vesicle formation (Fig. 1b)? Or does COPII somehow aid collagen export indirectly, without the need of a complete cage? How does the addition of ubiquitin change the geometry of the COPII coat? The new findings by Jin et al..$^{2}$ might aid in the development of a cell-free system for COPII-dependent packaging of collagen that would facilitate answers to such questions. Moreover, is Sec31 ubiquitination also relevant to the packaging of other large secreted macromolecules such as lipoproteins?

All these questions are relevant to our understanding not only of the fundamental mechanisms of cellular secretion but also of diseases where secretion (particularly of collagen) is defective because of gene mutations ${ }^{8}$. Moreover, manipulation of the $\mathrm{Cul}^{\mathrm{K}} \mathrm{Klhl}^{12}$ ubiquitination pathway might be used to increase collagen secretion from cells for applications in stem cell amplification, for growth of tissue components in regenerative medicine, or perhaps for ameliorating age-related degeneration of connective tissue.

\section{References}

1. Zanetti G, Pahuja KB, Studer S, Shim S, Schekman R. Nature Cell Biol. 2011; 14:20-28. [PubMed: 22193160]

2. Jin L, et al. Nature. 2012; XX:XX-XX.

3. Stagg SM, et al. Cell. 2008; 134:474-484. [PubMed: 18692470]

4. Fath S, Mancias JD, Bi X, Goldberg J. Cell. 2007; 129:1325-1336. [PubMed: 17604721]

5. Komander D. Biochem. Soc. Trans. 2009; 37:937-953. [PubMed: 19754430]

6. Saito K, et al. Cell. 2009; 136:891-902. [PubMed: 19269366]

7. Wilson DG, et al. J. Cell Biol. 2011; 193:935-951. [PubMed: 21606205]

8. De Matteis MA, Luini A. New Eng. J. Med. 2011; 365:927-938. [PubMed: 21899453] 

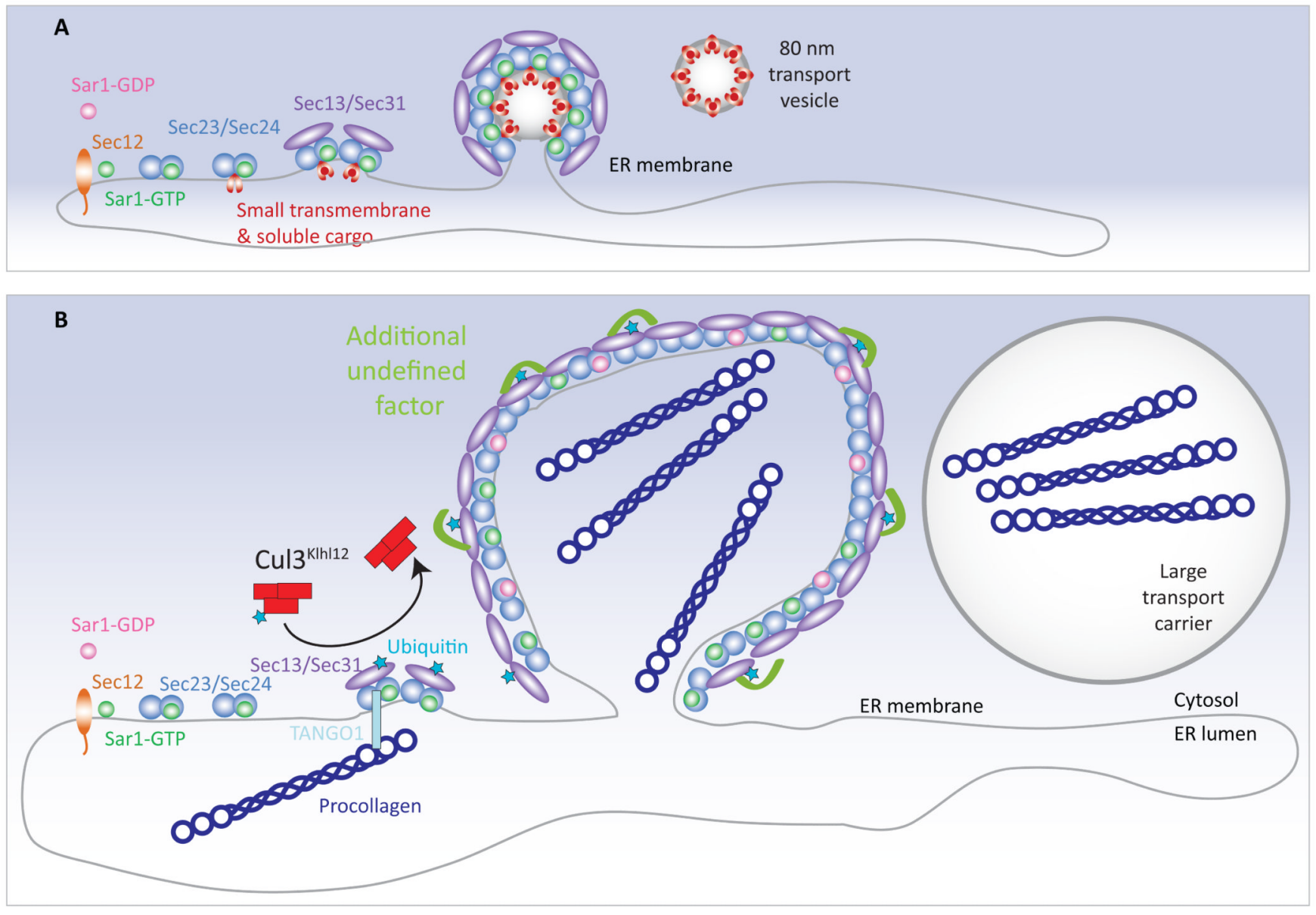

Figure 1. Big vesicles for collagen secretion

a, Soluble proteins targeted for secretion, together with small transmembrane proteins, are packaged at the endoplasmic reticulum into vesicles that are coated by the COPII protein cage. Proteins that will form the inner layer of the COPII coat associate in an ordered fashion, and then recruit the proteins $\operatorname{Sec} 13$ and Sec31, which will form the outer layer. This leads to membrane deformation and ultimately scission of 60-80 nm transport vesicles. b, Large proteins such as procollagen (the collagen precursor) do not fit within these typical vesicles. Jin et al. ${ }^{2}$ report that, to encapsulate these large cargoes, the enzyme Cul2 Klhl12 attaches one copy of the small protein ubiquitin to Sec31, and that this process facilitates collagen export. An additional unknown protein might further stabilize lateral Sec13-Sec31 interactions. Although it is not known whether collagen synthesis directly triggers $\mathrm{Cul} 2^{\mathrm{Klhl} 12}$ activity, the transmembrane protein TANGO1 — which couples collagen in the endoplasmic reticulum to the assembling coat on the cytosolic face - might have a role in the process. 